\title{
Management of mild traumatic brain injury-trauma energy level and medical history as possible predictors for intracranial hemorrhage
}

\author{
Tomas Vedin $^{1} \cdot$ Sebastian Svensson ${ }^{1} \cdot$ Marcus Edelhamre ${ }^{1} \cdot$ Mathias Karlsson $^{2} \cdot$ Mikael Bergenheim $^{3}$. \\ Per-Anders Larsson ${ }^{1}$
}

Received: 9 September 2017 / Accepted: 12 March 2018 / Published online: 17 March 2018

(c) The Author(s) 2018

\begin{abstract}
Purpose Head trauma is common in the emergency department. Identifying the few patients with serious injuries is time consuming and leads to many computerized tomographies (CTs). Reducing the number of CTs would reduce cost and radiation. The aim of this study was to evaluate the characteristics of adults with head trauma over a 1-year period to identify clinical features predicting intracranial hemorrhage.

Methods Medical record data have been collected retrospectively in adult patients with traumatic brain injury. A total of 1638 patients over a period of 384 days were reviewed, and 33 parameters were extracted. Patients with high-energy multitrauma managed with ATLS ${ }^{\text {TM }}$ were excluded. The analysis was done with emphasis on patient history, clinical findings, and epidemiological traits. Logistic regression and descriptive statistics were applied.

Results Median age was 58 years (18-101, IQR 35-77). High age, minor head injury, new neurological deficits, and low trauma energy level correlated with intracranial hemorrhage. Patients younger than 59 years, without anticoagulation or antiplatelet therapy who suffered low-energy trauma, had no intracranial hemorrhages. The hemorrhage frequency in the entire cohort was 4.3\% (70/1638). In subgroup taking anticoagulants, the frequency of intracranial hemorrhage was $8.6 \%$ (10/116), and in the platelet-inhibitor subgroup, it was $11.8 \%$ (20/169).

Conclusion This study demonstrates that patients younger than 59 years with low-energy head trauma, who were not on anticoagulants or platelet inhibitors could possibly be discharged based on patient history. Maybe, there is no need for as extensive medical examination as currently recommended. These findings merit further studies.
\end{abstract}

Keywords Brain injuries · Traumatic $\cdot$ Epidemiology $\cdot$ Practice guidelines as topic $\cdot$ Intracranial hemorrhage $\cdot$ Traumatic $\cdot$ S100B Calcium Binding Protein Beta Subunit

\section{Introduction}

During the twentieth century, patients with traumatic brain injury and loss of consciousness presenting in the emergency department were admitted for further observation. If they deteriorated during observation, computerized tomography (CT) was performed. Modern guidelines for managing traumatic brain injury in the emergency room have been utilized now for well over a decade and has alleviated
Tomas Vedin

tvedin@gmail.com

Sebastian Svensson

Sebastian.svensson@skane.se

Marcus Edelhamre

marcus_e@telia.com

Mathias Karlsson

mathias.karlsson@liv.se

Mikael Bergenheim

Mikael.bergenheim@liv.se
Per-Anders Larsson

P-a.larsson@telia.com

1 Clinical Sciences, Helsingborg, Lunds Universitet, Svartbrödragränden 3-5, 25187 Helsingborg, Sweden

2 Centralsjukshuset i Karlstad, Karolinska Institute, Rosenborgsgatan 9, 65230 Karlstad, Sweden

3 Centralsjukshuset i Karlstad, Umeå University, Rosenborgsgatan 9, 65230 Karlstad, Sweden 
clinical practice. When these guidelines are applied correctly, approximately $50 \%$ of the patients undergo head CTs [1].

Repetitive CT of the head may be related to adverse effects later in life. Even though modern CT machines are using less and less radiation, X-ray-induced cancer is something the clinician must take into account [2-5].

Modern guidelines are based on the previous epidemiological studies, which have shown a bimodal distribution of age, with the lowest incidence around 55 years of age. The highest incidence in the adult population is seen among young adults (predominantly males) and the elderly [6-12].

A randomized, controlled study from the same era showed that upfront CT when presenting in the emergency department was sufficient to rule out intracranial hemorrhage in most patients. They could be safely discharged [13]. North American studies have shown corresponding results, which have led to the establishment of the Canadian CT Head Rule and New Orleans Criteria [1, 14]. In the previous studies, the reported frequency of intracranial hemorrhage was $8 \%[1,15]$, Mortality ranged from 0.1 to $0.7 \%[6,15]$.

The common denominator for all degrees of traumatic brain injury is that the patient has sustained mechanical energy to the head from external physical force. However, trauma energy level varies. Both academic definitions and clinical guidelines are based primarily on level of consciousness, degree of head injury, and the temporal progression of level of consciousness. Neither the Canadian CT Head Rule, the New Orleans Criteria, nor the Scandinavian Neurotrauma Committee guidelines base decisions to dismiss patients on patient-stated trauma energy alone, suggesting that clinical status is the most important factor [1]. These guidelines use clinical features mostly as "rule-in criteria" for intracranial hemorrhage, giving patient history a subordinate role in relation with clinical status.

If patient history alone could be used as a "rule-out criterion" for intracranial hemorrhage, emergency department workloads and radiation levels could be reduced, resulting in both economic and public health benefits.

The aim of this study was to evaluate the characteristics of adults presenting with the chief complaint of head trauma over a 1-year period to identify clinical features predicting intracranial hemorrhage. The analysis was done with particular emphasis on patient history, clinical findings, and epidemiological traits.

\section{Methods}

The study was conducted as a retrospective analysis of medical records of patients presenting with head trauma at the emergency department of Helsingborg General Hospital. This hospital serves a geographic area of 350,000 people. The emergency department has approximately 72,000 visits annually. It has trauma surgeons, general surgeons, orthopedic surgeons, emergency medicine doctors, anesthesiologists, and otorhinolaryngologists. The nearest neurosurgical clinic is $40 \mathrm{~km}$ away. Multitrauma patients are managed according to ATLS ${ }^{\mathrm{TM}}$.

When presenting to the emergency department, patients were registered in an electronic patient registry. Its purpose was to keep track of room location and chief complaint. After extracting a list of all patients 18 years or older who registered with the chief complaint "head injury," we reviewed the medical records. Patients may have had other minor injuries as well, but were not initially classified as multitrauma. High-energy multitrauma patients $(n=647)$ were excluded to make the patient cohort more representative of the majority of emergency-room patients with head injury. Furthermore, the hospital manages multitrauma patients with a different algorithm, which we did not seek to evaluate. The review was performed on patients registered between November 11, 2013, and November 30, 2014, a total of 384 days.

The following parameters were manually extracted from medical records:

1. Age (years).

2. Gender $(\mathrm{m} / \mathrm{f})$.

3. Head CT performed (yes/no).

4. Head CT outcome (hemorrhage/no hemorrhage).

5. Admission to general hospital ward (yes/no).

6. Admission to intensive care unit (ICU) (yes/no).

7. Admission to neuro intensive care unit (neuro ICU) (yes/no).

8. Neurosurgical intervention (yes/no).

9. Degree of head injury (minimal, mild, moderate, and severe).

10. Level of consciousness using Reaction Level Scale 85 (RLS85) (1-8).

11. Level of consciousness using Glasgow Coma Scale (GCS) (15-3).

12. Blood pressure (systolic $\mathrm{mm} \mathrm{Hg}$ /diastolic $\mathrm{mm} \mathrm{Hg}$ ).

13. Pulse rate (beats/minute).

14. Size of pupils (mm).

15. Body weight (kilograms).

16. Height (meters).

17. Past medical history (yes/no).

18. Anticoagulant treatment (no/warfarin/noac/injection).

19. Platelet inhibitor treatment (no/aspirin/clopidogrel/ ticagrelor/other).

20. Other medication (yes/no).

21. Preexisting/new focal neurological deficits (yes/no).

22. Deterioration of neurological status during observation (yes/no).

23. S100B level $(\mu \mathrm{g} / \mathrm{L})$.

24. Nausea (yes/no).

25. Vomiting (yes/no).

26. Number of vomits (n). 
27. Amnesia, type and duration (yes/no, antegrade/retrograde, time hh:mm).

28. Loss of consciousness (yes/no).

29. Peritraumatic seizure (yes/no).

30. Posttraumatic headache (yes/no).

31. Increasing intensity of headache (yes/no).

32. Trauma energy levels (low, medium, and high).

33. Clinical signs of basal skull fracture (yes/no).

34. Orthostatic hypotension (yes/no).

35. Cardiac dysrhythmia (yes/no).

36. Time from injury to medical examination at the emergency department (hh:mm).

37. Influence of any or multiple drugs/alcohol (yes/no).

Level of trauma energy (see \#32 above) was interpreted on the basis of trauma mechanism, as follows:

- Low [1]: fall from less than $1 \mathrm{~m}$ or fewer than 5 stairs.

- Medium [1]: fall from 1 to $3 \mathrm{~m}$ or 5 or more stairs.

- High $\left(\mathrm{ATLS}^{\mathrm{TM}}\right)$ : fall from $3 \mathrm{~m}$ or more, motor vehicle accident at $70 \mathrm{~km} / \mathrm{h}$ or more with seatbelt, motor vehicle accident at $30 \mathrm{~km} / \mathrm{h}$ or more without seatbelt, any motorcycle accident, pedestrian hit by motor vehicle.

Minimal traumatic brain injury is trauma to the head without loss of consciousness and without any of the following criteria: amnesia, nausea, vomiting, vertigo, or focal neurological deficits [16].

Mild traumatic brain injury is an acute brain injury resulting from mechanical energy to the head from external physical forces. Operational criteria for clinical identification consist of the following:

1. One or more of the following: confusion or disorientation, loss of consciousness for $30 \mathrm{~min}$ or less, posttraumatic amnesia for less than $24 \mathrm{~h}$, and other transient neurological abnormalities such as focal signs, seizure, and intracranial lesion not requiring surgery

2. GCS score of $13-15$ either $30 \mathrm{~min}$ after injury or later upon presentation for healthcare [8].

Moderate traumatic brain injury is defined as a brain injury resulting in a loss of consciousness from $20 \mathrm{~min}$ to $6 \mathrm{~h}$ and a GCS score of 9-12 [17].

Severe traumatic brain injury is defined as a brain injury resulting in loss of consciousness for more than $6 \mathrm{~h}$ and a GCS score of 3-8 [17].

\section{Statistics}

Data were analyzed using SPSS version 21 for Mac. Q-Q plots and the Shapiro-Wilk formula were used to test for normal distribution. Central tendencies are presented as medians when skewed. Post hoc multivariate analysis was performed on all relevant and applicable parameters; this analysis was performed via univariate binomial logistic regression and the insertion of significant parameters $(p<0.4)$ in a multivariate logistic regression model. Another multivariate logistic regression was performed on the significant parameters $(p<0.05)$ of the first multivariate regression. Missing data were replaced by series median. Post hoc subgroup analysis with descriptive statistics was performed to ascertain the effect of different parameters on intracranial hemorrhage to determine the clinically significant age cutoff.

\section{Results}

The inclusion criteria were satisfied by 1,638 patients. Of the 1,638 patients, $456(27.8 \%)$ had minimal head injury, 922 (56.3\%) had mild head injury, $18(1.1 \%)$ had moderate head injury, $4(0.2 \%)$ had severe head injury, and 238 (14.5\%) were not assessable. Twelve patients were admitted to intensive care unit and 5 of these had neurosurgical intervention. Level of consciousness was classified according to the Scandinavian reaction level scale (RLS-85) in 1608 (98.2\%) cases. Only 47 cases $(2.7 \%)$ were classified according to GCS.

During the study period, 842 CTs were performed, representing $51.4 \%$ of the patients. Of these, 588 (69.8\%) were performed on patients with low-energy trauma. The median age of patients who did not undergo $C T$ was 48 years (18-101, IQR 28-67) and that of patients who underwent CT was 67 years (18-100, IQR 47-83). See Fig. 1 for age distribution of entire cohort.

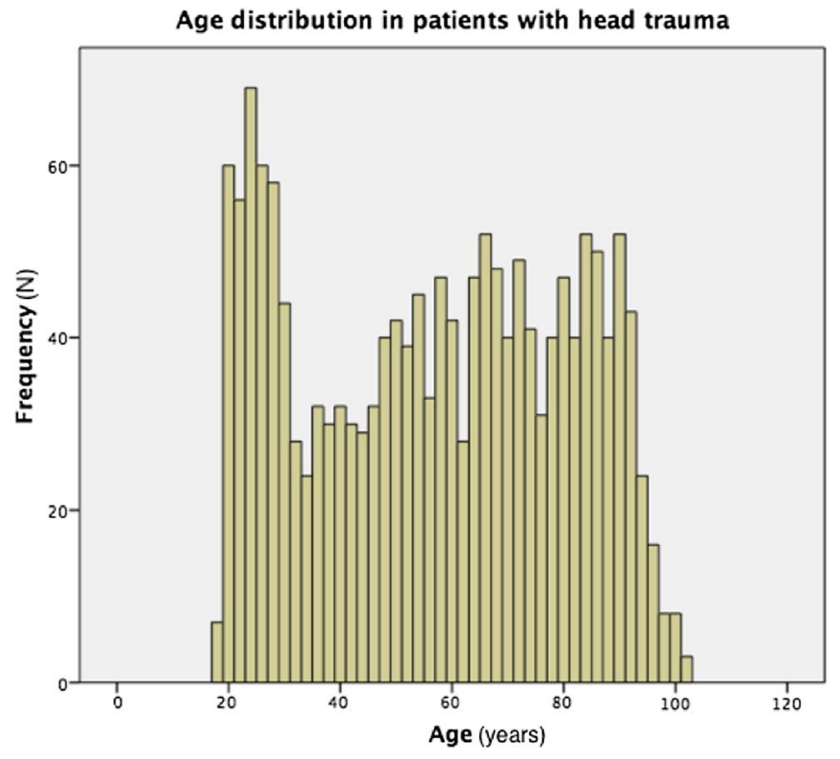

Age distribution in patients with trauma to the head over the entire study period

Fig. 1 Age distribution in patients with head trauma 
In multivariate logistic regression of 12 applicable predictor variables, only 4 were statistically significant: high age, minor head injury, new neurological deficits, and low trauma energy level (Table 1).

All patients with moderate or severe head injury underwent CT scan. Some parameters were excluded from analysis, because they occurred after head CT was performed or were not relevant to analysis of predictors for intracranial hemorrhage (admission to intensive care unit/neurointensive care unit/general ward, time at emergency room, time until admitted, and neurosurgical intervention). Some parameters

Table 1 Regression analysis of potential parameters predicting intracranial hemorrhage

\begin{tabular}{llll}
\hline Parameter & \multicolumn{2}{l}{$\mathrm{p}$-value } & \\
\cline { 2 - 4 } & $\begin{array}{l}\text { Univariate } \\
\text { regression }\end{array}$ & $\begin{array}{l}\text { Multivariate } \\
\text { regression } 1\end{array}$ & $\begin{array}{l}\text { Multivariate } \\
\text { Regression 2 }\end{array}$ \\
\hline Age & $<\mathbf{0 . 0 0 1}$ & $<\mathbf{0 . 0 0 1}$ & $<\mathbf{0 . 0 0 1}$ \\
Gender & $\mathbf{0 . 0 9 4}$ & $\mathbf{0 . 0 0 3}$ & 0.091 \\
Degree of head injury & $<\mathbf{0 . 0 0 1}$ & $<\mathbf{0 . 0 0 1}$ & $<\mathbf{0 . 0 0 1}$ \\
Level of consciousness & $\mathbf{0 . 0 1 6}$ & 0.469 & $\mathrm{n} / \mathrm{a}^{* *}$ \\
$\quad$ RLS) & & & \\
Past illness & $\mathbf{0 . 0 1 5}$ & 0.792 & $\mathrm{n} / \mathrm{a}^{* *}$ \\
Anticoagulation treatment & $\mathbf{0 . 0 1 3}$ & 0.214 & $\mathrm{n} / \mathrm{a}^{* *}$ \\
Platelet inhibitor & $<\mathbf{0 . 0 0 1}$ & 0.111 & $\mathrm{n} / \mathrm{a}^{* *}$ \\
Current medication & 0.616 & $\mathrm{n} / \mathrm{a}^{*}$ & $\mathrm{n} / \mathrm{a}^{* *}$ \\
Preexisting neurological & 0.957 & $\mathrm{n} / \mathrm{a}^{*}$ & $\mathrm{n} / \mathrm{a}^{* *}$ \\
$\quad$ deficits & & & \\
New neurological deficits & $<\mathbf{0 . 0 0 1}$ & $\mathbf{0 . 0 0 7}$ & $<\mathbf{0 . 0 0 1}$ \\
Trauma energy level & $<\mathbf{0 . 0 0 1}$ & $<\mathbf{0 . 0 0 1}$ & $<\mathbf{0 . 0 0 1}$ \\
Intoxication & $\mathbf{0 . 0 3 4}$ & 0.194 & $\mathrm{n} / \mathrm{a}^{* *}$ \\
\hline
\end{tabular}

Bold values indicate statistically significant

*Value is marked $\mathrm{n} / \mathrm{a}$ when significance lever in the previous analysis was too low for inclusion in the next statistical analysis $(p<0.4)$

$* *$ Value is marked $\mathrm{n} / \mathrm{a}$ when significance lever in the previous analysis was too low for inclusion in the next statistical analysis $(p<0.05)$ were excluded due to $>30 \%$ missing values [level of consciousness (GCS), blood pressure (systolic and diastolic), pulse rate, weight, height, size of pupils, s100b level, loss of consciousness, nausea, vomiting, number of vomits, amnesia type and duration, seizures, headache on admission, worsening headache, orthostatic hypotension, cardiac dysrhythmia, time from trauma to medical examination and signs of basal skull fracture].

In the 58 years or younger subgroup $(n=826 / 1638$ $[50.4 \%]$ ), no intracranial hemorrhage was found in patients with low-energy trauma. When not stratifying this age cohort according to intracranial hemorrhage, patients with all degrees of head injury were found. No patients in this subgroup had anticoagulants or platelet inhibitors. Of the patients with intracranial hemorrhage, two were missing the trauma-energy parameter (Table 2).

Further analysis of these two patients revealed that both had sustained the head injury while intoxicated and were unable to report the trauma mechanism. One of these patients, from the 18-39 age group, was injured during a fist fight, and the other, from the 40-58 age group, was injured in unclear circumstances. Both had ongoing intravenous substance abuse, and both presented to the emergency room several days after trauma.

353 patients $(21.6 \%)$ were admitted to the surgical ward due to head trauma. Seven $(0.4 \%)$ patients were cared for at the ICU and five $(0.3 \%)$ patients underwent neurosurgical intervention and received care at the neuro ICU. 70/1638 (4.3\%) patients had intracranial hemorrhage. Of the patients undergoing CT scan, $8.3 \%$ had intracranial hemorrhage. $76 \%$ of patients sustained low-energy force. $6 \%$ sustained medium energy trauma, $1.5 \%$ sustained high-energy trauma, and in $17.5 \%$ of cases, it was not assessable.

The distribution of intracranial hemorrhage was 45/70 $(64.3 \%)$ in males and 25/70 (35.7\%) in females (Table 3).

Of the 1638 patients, 1469 (89.7\%) had no thrombocyte aggregation inhibitor, and 169 (11.3\%) had some sort of thrombocyte aggregation inhibitor, where $75 \mathrm{mg}$ ASA
Table 2 Age, energy level, and frequency of intracranial hemorrhage

\begin{tabular}{|c|c|c|c|c|c|}
\hline & \multicolumn{4}{|l|}{ Age (years)* } & \multirow[t]{2}{*}{$N$ Total } \\
\hline & $18-39$ & $40-58$ & $59-79$ & $80-101$ & \\
\hline & \multicolumn{5}{|c|}{$N$ Intracranial hemorrhage $/ N$ total cohort } \\
\hline \multicolumn{6}{|c|}{ Energy level } \\
\hline Low & $0 / 328$ & $0 / 251$ & $16 / 349$ & $27 / 323$ & 1251 \\
\hline Medium & $0 / 47$ & $3 / 28$ & $5 / 17$ & $2 / 2$ & 94 \\
\hline High & $2 / 11$ & $1 / 3$ & $0 / 0$ & $2 / 2$ & 16 \\
\hline $\mathrm{n} / \mathrm{a}$ & $1 / 93$ & $1 / 65$ & $5 / 75$ & $5 / 44$ & 277 \\
\hline \multicolumn{6}{|c|}{$N$ patients with intracranial hemorrhage } \\
\hline & $3 / 479(0.6 \%)$ & $5 / 347(1.4 \%)$ & $26 / 441(5.9 \%)$ & $36 / 371(9.7 \%)$ & 70 \\
\hline
\end{tabular}

*Group cutoffs were selected at middle age (40 years), first occurrence of intracranial hemorrhage in lowenergy trauma group (59 years), and age when intracranial hemorrhages are much more common ( 80 years) 
Table 3 Intracranial hemorrhage, age, and gender

\begin{tabular}{llllll}
\hline Gender and age & Hemorrhages & \multicolumn{4}{l}{ Energy of trauma } \\
\cline { 3 - 6 } & & Low & Medium & High & n/a \\
\hline Male $(N$ hemorrhages $)$ & 45 & 26 & 9 & 2 & 8 \\
Mean age [years $( \pm$ SD $)]$ & $74( \pm 12.96)$ & $79( \pm 10.16)$ & $69( \pm 10.54)$ & $67( \pm 18.38)$ & $67( \pm 15.53)$ \\
Female $(N$ hemorrhages $)$ & 25 & 17 & 1 & 2 & 5 \\
Mean age [years $( \pm$ SD $)]$ & $77( \pm 19,21)$ & $82( \pm 10.7)$ & 45 & $58,5( \pm 30.41)$ & $73( \pm 31.56)$ \\
\hline
\end{tabular}

Table 4 S100b levels and intracranial hemorrhage

\begin{tabular}{lll}
\hline s100b Level & \multicolumn{2}{l}{$\begin{array}{l}\text { Intracranial } \\
\text { hemorrhage }\end{array}$} \\
\cline { 2 - 3 } & Yes & No \\
\hline$>0.1$ umol/1 & 7 & 102 \\
$<0.1$ umol $/ 1$ & 1 & 87 \\
Not measured & 62 & 1379 \\
\hline
\end{tabular}

accounted for most cases (148/1638). The other 21 patients had treatment with Clopidrogrel $75 \mathrm{mg}$, Ticagrelor, or a combination of these.

Moreover, 1,505 (91.9\%) had no anticoagulation, 119 (7.3\%) had warfarin, and $18(1.1 \%)$ had oral anticoagulants or injections of low-molecular-weight heparin with varying dosage.

Of the patients, $23.3 \%$ were intoxicated with alcohol, and $25 \%$ in total were intoxicated with some kind of drug or a combination of drugs.

Moreover, 92\% were assessed using the Reaction Level Scale 1 (RLS1) on their arrival to the emergency department. Of these, $28 \%$ had deteriorating mental status (i.e., went from RLS1 to RLS2, etc.) during their emergencydepartment stay. Observed deterioration of mental status was not associated with higher frequency of intracranial hemorrhage.

Patients without anticoagulants and platelet inhibitors who suffered head trauma had a 3\% (40/1351) incidence of intracranial hemorrhage. The hemorrhage frequency in the entire population was $4.3 \%$ (70/1638). Among patients on anticoagulants, the frequency of intracranial hemorrhage was $8.6 \%$ (10/116), and it was $11.8 \%$ (20/169) among those on platelet inhibitors (Table 3).

S100b was measured in $198 / 1638$ cases. Sensitivity was $87.5 \%$, specificity was $46.0 \%$, the negative predictive value was 0.989 , and the positive predictive value was 0.064 (Table 4).

\section{Discussion}

This study showed that high age, male gender, low trauma energy, low degree of head injury, and new neurological deficits were correlated with intracranial hemorrhage. These correlations are in accordance with the previous studies and do not reveal any new clinically significant information $[1,14]$. To focus on the patient group presenting with the chief complaint 'head trauma' and to identify the common traits for this group of patients, all high-energy multitrauma patients were excluded.

Because of the results of the logistic regression analysis, subgroup analysis was performed. This yielded the large group of patients 58 years or younger who did not have any intracranial hemorrhage when trauma energy was low and who did not take anticoagulation and/or antiplatelet therapy. However, because of unreliable patient history with regard to trauma energy in two of the cases, hemorrhages were found also in this age cohort. The in-depth analysis of these two cases revealed that trauma energy level could not be determined.

The validity of this subgroup finding is debatable because of missing data, but it certainly merits further prospective studies. A retrospective analysis of this kind has two main issues of bias to take into account. First, the data are entered into medical records by different physicians who all make individual and slightly different assessments. Second, the medical record data were interpreted by another person and converted to categorical data. We tried to reduce this bias using a rigorous set of rules for how data should be categorized during data collection. We are currently planning a study to evaluate the validity of the present findings, where data will be prospectively recorded and categorized upon collection to minimize bias.

In the Canadian CT Head Rule, medium trauma energy is used as a "rule-in criterion" for head CT, regardless of clinical presentation. However, low trauma energy is not used as a "rule-out criterion." In the case of low trauma energy, signs and symptoms guide the decision of whether to use CT [1]. In the New Orleans Criteria and Scandinavian Neurotrauma Committee guidelines, trauma energy is not used as a predictor at all $[14,18]$. In all three guidelines, level of consciousness is used as a predictor and moderate head injury prompts CT. This is not contradicted by the present study (patients with all degrees of head injury were found both in the 58 years or younger subgroup and in the entire cohort), but it suggests that a higher degree of head injury does not have to be a "rule-in criterion" if other criteria, such as the characteristics of the aforementioned subgroup, are met. 
The present study indicates the possibility of ruling out intracranial hemorrhage in some patients based on patient history alone, regardless of clinical status.

The epidemiological analysis shows slightly higher average age compared to the previous studies However, this trend was demonstrated by Roozenbeek et al. in a large meta-analysis [19]. This indicates that the trend has not yet culminated. The distribution between degrees of head injury is largely the same as in studies from the past decade $[9,12$, 20]. Because of these findings, which are in line with those of the previous studies, we conclude that the present study has a relevant patient cohort with statistical validity.

The present study indicates a higher frequency of intracranial hemorrhage in patients with antiplatelet medication than in patients with anticoagulants. These results are in accordance with the previous studies by Nishijima et al. [21]. Both our findings and those of Nishijima et al. indicate that guidelines should give as much attention to antiplatelet therapy as to anticoagulation therapy, which is not the case today [1].

The study design, a retrospective review of medical records, has certain limitations. Therefore, one has to be careful not to jump to conclusions based on its results. However, if these findings are reproducible in a prospective study, almost half of the adult patients with traumatic head injury may be safely discharged by the triage nurse based on patient history alone. This would eliminate further medical examination and irradiation and warrants further evaluation in a prospective study, where patient history and trauma energy play a central role in management.

\section{Conclusion}

This retrospective study demonstrates that patients younger than 59 years, with low-energy head trauma who were not on anticoagulants or platelet inhibitors could have been discharged after complete patient history and may not have needed as extensive medical attention as currently recommended and here given. These findings merit further studies.

Acknowledgements This work was supported by the Gorthon Foundation, Helsingborg.

\section{Compliance with ethical standards}

Ethics approval The study was approved by the Regional Ethical Review Board in Lund. It was conducted in accordance with Helsinki declaration. Specific national laws were observed.

Conflicts of interest Authors declare no conflicts of interest.

Open Access This article is distributed under the terms of the Creative Commons Attribution 4.0 International License (http://creativeco mmons.org/licenses/by/4.0/), which permits unrestricted use, distribution, and reproduction in any medium, provided you give appropriate credit to the original author(s) and the source, provide a link to the Creative Commons license, and indicate if changes were made.

\section{References}

1. Stiell IG, Wells GA, Vandemheen K, Clement C, Lesiuk H, Laupacis A, McKnight RD, Verbeek R, Brison R, Cass D, Eisenhauer ME, Greenberg G, Worthington J. The Canadian CT Head Rule for patients with minor head injury. Lancet. 2001;357:1391-6.

2. Smith-Bindman R, Lipson J, Marcus R, Kim KP, Mahesh M, Gould R, Berrington de Gonzalez A, Miglioretti DL. Radiation dose associated with common computed tomography examinations and the associated lifetime attributable risk of cancer. Arch Intern Med. 2009;169:2078-86.

3. Brenner DJ. Should we be concerned about the rapid increase in CT usage? Rev Environ Health. 2010;25:63-8.

4. Webster NJ, Moore N, Stewart F. Reducing unnecessary head computed tomography in mild traumatic brain injury. Adv Emerg Nurs J. 2017;39:300-8.

5. Goldberg J, McClaine RJ, Cook B, Garcia VF, Brown RL, Crone $\mathrm{K}$, Falcone RA. Use of a mild traumatic brain injury guideline to reduce inpatient hospital imaging and charges. J Pediatr Surg. 2011;46:1777-83.

6. Andersson EH, Bjorklund R, Emanuelson I, Stalhammar D. Epidemiology of traumatic brain injury: a population based study in western Sweden. Acta Neurol Scand. 2003;107:256-9.

7. Bruns J, Hauser WA. The epidemiology of traumatic brain injury: a review. Epilepsia. 2003;44:2-10.

8. Carroll L, Cassidy JD, Peloso P, Borg J, Von Holst H, Holm L, Paniak C, Pépin M. Prognosis for mild traumatic brain injury: results of the WHO Collaborating Centre Task Force on Mild Traumatic Brain Injury. J Rehabil Med. 2004;36:84-105.

9. Langlois JA, Rutland-Brown W, Wald MM. The epidemiology and impact of traumatic brain injury: a brief overview. J Head Trauma Rehabil. 2006;21:375-8.

10. Rutland-Brown W, Langlois JA, Thomas KE, Xi YL. Incidence of traumatic brain injury in the United States, 2003. J Head Trauma Rehabil. 2006;21:544-8.

11. Tagliaferri F, Compagnone C, Korsic M, Servadei F, Kraus J. A systematic review of brain injury epidemiology in Europe. Acta Neurochir (Wien). 2006;148:255-68 (discussion 268).

12. Corrigan JD, Selassie AW, Orman JAL. The epidemiology of traumatic brain injury. J Head Trauma Rehabil. 2010;25:72-80.

13. af Geijerstam J, Oredsson S, Britton M. OCTOPUS-observation or computed tomography of mild head injury in Sweden: a randomised clinical trial concerning effects and costs. Crit Care. 2005;9:P281.

14. Haydel MJ, Preston CA, Mills TJ, Luber S, Blaudeau E, DeBlieux PMC. Indications for computed tomography in patients with minor head injury. N Engl J Med. 2000;343:100-5.

15. af Geijerstam J-L, Britton M. Mild head injury-mortality and complication rate: meta-analysis of findings in a systematic literature review. Acta Neurochirurgica. 2003;145:843-50.

16. Ingebrigtsen T, Romner B, Kock-Jensen C. Scandinavian guidelines for initial management of minimal, mild, and moderate head injuries. Scandinavian Neurotrauma Committee J Trauma. 2000;48:760-6.

17. Network TBI. Traumatic brain injury network. $2016 \mathrm{http} / / / \mathrm{www}$. traumaticbraininjury.com/symptoms-of-tbi/.

18. Unden J, Ingebrigtsen T, Romner B. Scandinavian guidelines for initial management of minimal, mild and moderate head injuries 
in adults: an evidence and consensus-based update. BMC Med. 2013;11:50.

19. Roozenbeek B, Maas AI, Menon DK. Changing patterns in the epidemiology of traumatic brain injury. Nat Rev Neurol. 2013;9:231-6.

20. Andriessen TMJC., Horn J, Franschman G, van der Naalt J, Haitsma I, Jacobs B, Steyerberg EW, Vos PE. Epidemiology, severity classification, and outcome of moderate and severe traumatic brain injury: a prospective multicenter study. J Neurotrauma. 2011;28:2019-31.

21. Nishijima DK, Offerman SR, Ballard DW, Vinson DR, Chettipally UK, Rauchwerger AS, Reed ME, Holmes JF. Immediate and delayed traumatic intracranial hemorrhage in patients with head trauma and preinjury warfarin or clopidogrel use. Ann Emerg Med. 2012;59:460-8. e7. 